\title{
TWO DIMENSIONAL SINK FLOW OF A VISCOUS, HEAT-CONDUCTING, COMPRESSIBLE FLUID; CYLINDRICAL SHOCK WAVES*
}

\author{
BY \\ T. YAO-TSU WU \\ California Institute of Technology
}

Introduction. The problem of the steady cylindrical source-type or sink-type flow has been of interest to fluid-dynamicists for several reasons. First, it is known that the corresponding problem of an inviscid compressible fluid has an exact solution containing a limit line of rather special type, namely, the sonic circle [2]. To the exterior of this circle the solution has two branches of values, one has its stagnation point at infinity (subsonic branch) and the other starts with maximum velocity at infinity (supersonic branch). Both of these two branches terminate at the limit line with infinite velocity gradient. Therefore the viscous and heat-conductive effects are expected to play an important role in continuing the solution further inward. Second, because of its cylindrical symmetry, this problem is one of the few nonlinear flows in more than one dimension for which there is only one independent variable, the radial distance. Consequently, the equations are simple enough to allow a unified discussion of the various effects. These are perhaps the reasons why this problem has attracted the attention of several authors $[3,4,5]$.

In the first part of this investigation the Navier-Stokes equations are given for the cylindrical sink flow of a viscous, heat-conducting, perfect gas. Then, with some simplifying assumptions, the qualitative properties of the solutions are discussed in detail for the case of large Reynolds number $R e$. The definition of $R e$ is $R e=\rho_{1} a_{1} r_{1} / \mu_{1}$, where $r=r_{1}$ locates the inviscid sonic circle with sonic speed $a_{1}$, and $\rho_{1}, \mu_{1}$ are the fluid density and viscosity coefficient at $r=r_{1}$. These basic properties of the solutions thus comprehended serve as a useful guide in our final calculation of the solution.

In the second part of this paper the detailed calculation of the solution is carried out for the case of large $R e$. It is found that there is no single expression available for the solution uniformly valid in the entire flow region. The calculation is then performed in three different regions characterized by the length $r_{1}$ and the parameter Re. For $r>r_{1}+O\left(R e^{-2 / 3}\right)$ the approximate solution is obtained by using the PLK method**. The result fails to be a good approximation for $r$ too close to $r_{1}$. For $\left|r-r_{1}\right|<O\left(R e^{-2 / 3}\right)$, a different similarity rule for the variables leads to a system of cylindrical transonic equations which governs the flow across the sonic region. These equations can be integrated analytically for each of the different order terms. The result shows that the solutions belonging to the supersonic branch all contain cylindrical shock-type flow in this transonic region. In other words, these solutions gradually deviate away from the inviscid supersonic branch, reach a minimum near $r=r_{1}$ and then approach asymptotically to the viscous subsonic branch. It is also found that the shock strength is of $O\left(R e^{-1 / 3}\right)$ while the shock-thickness is of $O\left(R e^{-2 / 3}\right)$, results which are quite different

*Received Sept. 10, 1954. This paper is the slightly condensed version of a Hydrodynamics Laboratory report [1] of California Institute of Technology. The work was sponsored by the Office of Naval Research under the Contract Number N6onr-24420 (NR 062-059).

**This terminology was introduced by Prof. H. S. Tsien in a series of seminars, held in California Institute of Technology in 1954, in which the method due to Poincare-Lighthill-Kuo was discussed. 
from those of the plane normal shock. Within this region, the thermodynamic variables satisfy the isentropic relation up to the order $O\left(R e^{-1 / 3}\right)$ but deviate from it by a quantity of $O\left(R e^{-2 / 3}\right)$. The approximate solution for $r<r_{1}-O\left(R e^{-2 / 3}\right)$ is subsequently carried out. Finally, the entropy variation of the fluid, and the effect due to variation in viscosity coefficients, are discussed.

The corresponding source flow problem was previously solved, using a numerical method, by Sakurai [4]; a qualitative investigation on this problem was later elaborated on many points by Levey [5], by making use of some conventional methods in nonlinear differential equations. In the latter work, the orders of magnitude of many flow quantities of interest were estimated. The present investigation on the sink flow is not merely a special case of the cylindrical flow other than the source type, but also presents an improved method which is more powerful than those used in the previous works (e.g. Ref. $[3,4,5])$. The PLK-method applied to the outer region yields a set of reliable boundary values for the transonic region and thus enables all flow quantities of interest to be calculated quantitatively in all regions.

The author thanks Prof. H. S. Tsien for suggesting the problem and Profs. M. S. Plesset and C. R. DePrima for their assistance on many points.

1. The fundamental equations. Here we are concerned with the two-dimensional sink flow of a viscous, compressible, heat-conducting fluid with polar symmetry. The only independent variable is the radial distance $r$ from the origin. The radial velocity, $u$, is the only velocity component and is always negative for $\operatorname{sink}$ flow. Let $p, \rho, T, \mu$, $\mu^{\prime}, \lambda, R, C_{v}, C_{p}$ denote respectively the pressure, density, absolute temperature, coefficients of shear and bulk viscosity, heat conductivity, gas constant, specific heats at constant volume and pressure. The momentum equation is

$$
\rho u \frac{d u}{d r}=-\frac{d p}{d r}+\frac{d}{d r}\left[2 \mu \frac{d u}{d r}+\frac{2}{3}\left(\mu^{\prime}-\mu\right) \frac{1}{r} \frac{d}{d r}(r u)\right]+2 \mu \frac{d}{d r}\left(\frac{u}{r}\right)
$$

and the energy equation is

$$
\text { pur } \frac{d}{d r}\left(\frac{u^{2}}{2}+C_{p} T\right)=\frac{d}{d r}\left\{r\left[\lambda \frac{d T}{d r}+\mu \frac{d u^{2}}{d r}+\frac{2}{3}\left(\mu^{\prime}-\mu\right)\left(\frac{1}{2} \frac{d u^{2}}{d r}+\frac{u^{2}}{r}\right)\right]\right\} .
$$

The continuity equation can be written in the following form if $m$ denotes the sink strength,

$$
2 \pi \rho u r=-m .
$$

The equation of state is assumed to be that of a perfect gas,

$$
p=R \rho T \text {. }
$$

Equations $(1.1$ - 1.4) are a system of nonlinear differential equations for four variables $u, p, \rho$ and $T$ if $\mu, \mu^{\prime}, \lambda$ and $C_{p}$ are known functions of $T$.

To reduce the equations to nondimensional form, the following nondimensional quantities are introduced:

$$
\begin{gathered}
r^{*}=r / r_{1}, \quad \eta=\log r^{*}, \quad w=-u / a_{1}, \quad \theta=T / T_{1}=\left(a / a_{1}\right)^{2}, \\
p^{*}=p / p_{1}, \quad \rho^{*}=\rho / \rho_{1}, \quad \mu^{*}=\mu / \mu_{1}, \quad \mu^{*}=\mu^{\prime} / \mu_{1},
\end{gathered}
$$


where quantities with the subscript 1 are fictitious quantities which would occur at the local Mach number unity for nonviscous and non-heat-conducting gas. Thus, with $\gamma$ equal to the ratio of specific heats, assumed constant throughout, the sonic speed $a_{1}$ at $r=r_{1}$ is given by

$$
a_{1}^{2}=\gamma p_{1} / \rho_{1} \quad \text { and } \quad 2 \pi \rho_{1} a_{1} r_{1}=m .
$$

The continuity equation then becomes

$$
\rho^{*} w r^{*}=1 .
$$

Here $w$ is always positive for sink flow. The equation of state is now

$$
p^{*}=\theta \rho^{*} \text {. }
$$

Eliminating $p$ and $\rho$ in Eq. (1.1) by using (1.3) and (1.4), and introducing the nondimensional quantities, with $\eta=\log r^{*}$ as the independent variable, we obtain

$$
\begin{aligned}
\frac{d w}{d \eta}+\frac{1}{\gamma}\left[\frac{d}{d \eta}\left(\frac{\theta}{w}\right)\right. & \left.-\frac{\theta}{w}\right] \\
& =-2 \alpha^{*}\left\{\mu^{*}(1+k)\left(\frac{d^{2} w}{d \eta^{2}}-w\right)+\left[(1+k) \frac{d w}{d \eta}+k w\right] \frac{d \mu^{*}}{d \eta}\right\},
\end{aligned}
$$

where

$$
\alpha^{*} \equiv(R e)^{-1}=\frac{\mu_{1}}{\rho_{1} a_{1} r_{1}}=\frac{2 \pi \mu_{1}}{m}, \quad \mu^{\prime}=(1+3 k) \mu .
$$

$\alpha^{*}$ thus denotes the inverse of the Reynolds number and will be considered much smaller than unity throughout this paper; whereas $k$ expresses the relation between the two viscosity coefficients. Stokes' assumption on the value of $\mu^{\prime}$ states that

$$
\mu^{\prime}=0 \quad \text { or } \quad k=-1 / 3 .
$$

As this assumption does not agree with observations for many kinds of fluid [6], condition (1.11) will not be imposed on the final calculation of the flow field.

By using again Eq. (1.3), the energy equation (1.2) can be integrated once to yield the following nondimensional form

$$
\frac{w^{2}}{2}+\frac{\theta}{\gamma-1}+\alpha^{*} \mu^{*}\left[\frac{\sigma^{-1}}{\gamma-1} \frac{d \theta}{d \eta}+(1+k) \frac{d w^{2}}{d \eta}+2 k w^{2}\right]=\frac{\gamma+1}{2(\gamma-1)},
$$

where $\sigma$ denotes the Prandtl number

$$
\sigma=C_{p} \mu / \lambda .
$$

The integration constant on the right hand side of Eq. (1.12) is chosen as shown above so that the limit solution for vanishing viscosity agrees at large $r$ with that of a nonviscous iso-energetic flow.

Equations (1.9) and (1.12) are the two equations for two unknowns $w$ and $\theta$. The boundary conditions for them can be determined by requiring that they tend to their respective inviscid solutions as $\eta \rightarrow \infty$ so that these two solutions can appropriately be compared later.

1.1 The inviscid solution. The solution for sink flow of a compressible inviscid gas can be literally obtained by putting $\alpha^{*}=0$ in the above equations without justifying 
the validity of such a simplification. The solution of this reduced system of equations is known to be

$$
\frac{r}{r_{1}}=e^{\eta}=w^{-1}\left(\frac{\gamma+1}{2}-\frac{\gamma-1}{2} w^{2}\right)^{-1 /(\gamma-1)}
$$

and

$$
\theta=\frac{\gamma+1}{2}-\frac{\gamma-1}{2} w^{2}, \quad p^{*}=\left(\rho^{*}\right)^{\gamma}=\theta^{\gamma /(\gamma-1)} .
$$

The value of $r_{1}$ can be expressed in terms of stagnation state as

$$
r_{1}=\frac{m}{2 \pi a_{1} \rho_{1}}=\frac{m}{2 \pi a_{0} \rho_{0}}\left(\frac{\gamma+1}{2}\right)^{(1 / 2)(\gamma+1) /(\gamma-1)}
$$

where $a_{0}^{2}=\gamma p_{0} / \rho_{0}$ and $p_{0}, \rho_{0}$ are the isentropic stagnation pressure and density. Equation (1.15) simply states the iso-energetic and isentropic relations.

This inviscid solution $w(r)$ given by Eq. (1.14) is plotted in Fig. 1. It gives no solution for $r<r_{1}$; but for $r>r_{1}, w$ is a double-valued function of $r$. On one branch $w$ tends to

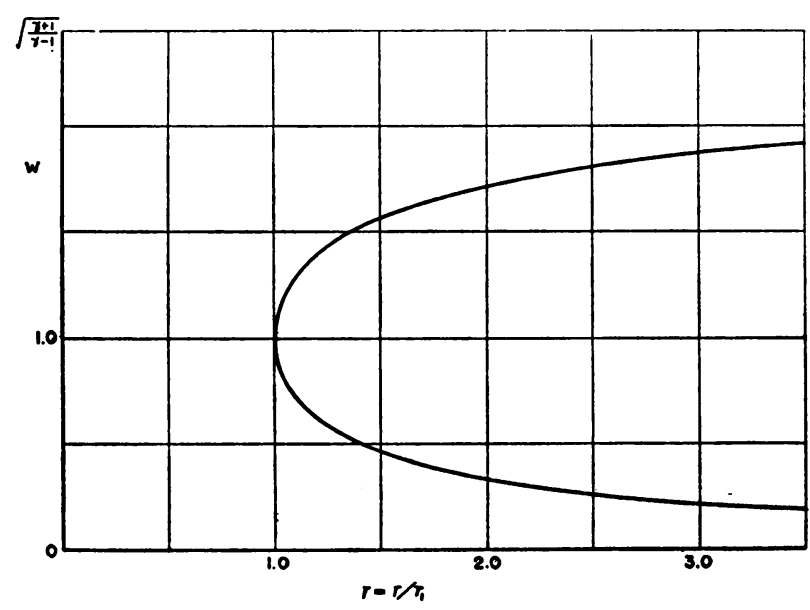

FIg. 1. Graph of inviscid solution.

zero so that thermodynamic variables tend to their stagnation values as $r \rightarrow \infty$; on the other branch $w$ tends to the maximum speed attainable $[(\gamma+1) /(\gamma-1)]^{1 / 2}$, and the thermodynamic variables tend to zero as $r \rightarrow \infty$. They will be designated as subsonic and supersonic branch respectively. Both of these branches terminate at $r=r_{1}$ with sonic speed (at which the fluid speed equals the local speed of sound). The slope of the curve $w(r)$,

$$
\frac{d w}{d r^{*}}=\frac{2}{\gamma+1} w^{2}\left(w^{2}-1\right)^{-1}\left(\frac{\gamma+1}{2}-\frac{\gamma-1}{2} w^{2}\right)^{\gamma /(\gamma-1)}
$$

is much smaller than unity for $r \gg r_{1}$ on both branches and consequently viscous effects become comparatively unimportant there. But as $r \rightarrow r_{1}, w \rightarrow 1, d w / d r$ becomes numerically unbounded, and thus the viscous force near $r=r_{1}$ should play a role as significant as those of inertia and pressure forces. 
Now this inviscid solution will be used as a guide to study the sink flow of a real fluid governed by Eqs. (1.9) and (1.12) for large values of $R e$, in the sense that it is assumed that the limit of the viscous solutions for vanishing viscosity approaches the inviscid solution as $r \rightarrow \infty$, for both subsonic and supersonic branches. By continuing these viscous solutions backward in $r$ where viscous effects become more and more prominent, it is expected that the real fluid, affected by viscosity and heat conduction, will flow across this fictitious sonic circle, which is a limit line when viscosity is neglected.

It may be remarked here that the equations for source flow of real fluid can be obtained from (1.9) and (1.12) by changing the sign of the terms with factor $\alpha^{*}$ if $w$ again represents the absolute value of the radial speed, normalized relative to $a_{1}$ [5]. Hence the inviscid solutions for source and sink flow are identical, but their respective viscous solutions will be shown later to have quite different features for all $r$.

\section{Properties of the solution curves.}

2.1. Approximate differential equation in the "phase space". In order to study the qualitative properties of the solution curves, several assumptions will be introduced in this section to simplify the analysis while most of the important features of the original system will still be maintained. The Prandtl number, $\sigma$, is assumed constant because $\mu$ and $\lambda$ have almost the same dependence on temperature. In this section, $\mu$ is also taken to be constant so that $\mu^{*}=1$. When the complete solution is calculated later, these assumptions introduced here become unnecessary.

Equation (1.12) can be integrated when the Prandtl number

$$
\sigma=\frac{1}{2}\left(1+4 \alpha^{*} k\right) /(1+k)
$$

(under Stokes' assumption, $k=-1 / 3$, then $\sigma=3 / 4-\alpha^{*}$ ), and the final integral is

$$
\theta-\left[\frac{\gamma+1}{2}-\frac{\gamma-1}{2}\left(1+4 \alpha^{*} k\right) w^{2}\right]=E \exp \left(-\sigma \eta / \alpha^{*}\right)=E\left(\frac{r}{r_{1}}\right)^{-\sigma / \alpha^{*}},
$$

where $E$ is the integration constant. The value chosen above for $\sigma$ is actually not far from experimental data ( $\sigma \doteq 0.72$ for air at standard condition). As $\sigma$ only appears in the coefficient of the derivative in Eq. (1.12), it follows from the theory of differential equations [Ref. 7, p. 142] that the solutions and all their derivatives will be continuous in $\sigma$ for $w>0,-\infty<\sigma<\infty$. Thus the assumption of choosing this particular value of $\sigma$ would merely lead to simplification of analysis rather than any material change of the solutions. If we further require by physical argument that the deviation from the isoenergetic relation expressed by the term with the arbitrary constant $E$ shall not overwhelm the left hand side terms for $r<r_{1}$, we may assume that $E=0$. This restriction, however, can again be relaxed when the complete solution is discussed later. It will then be shown that $E$ is indeed of the order $0\left(\alpha^{*}\right)$. Thus the particular solution with $E=0$ would still provide a good approximation to the complete solution.

Substituting Eq. (2.2) with $E=0$ into (1.9) and eliminating the explicit dependence on $\eta$ by the substitution

$$
V=-\frac{d w}{d \eta}=-r \frac{d w}{d r}
$$

we obtain

$$
\alpha w^{2} V \frac{d V}{d w}+V\left[1-(1-a \alpha) w^{2}\right]-w\left\{1-[\beta+(a-1) \alpha] w^{2}\right\}=0,
$$


where

$$
\alpha=\frac{4 \gamma}{\gamma+1}(1+k) \alpha^{*}, \quad \beta=\frac{\gamma-1}{\gamma+1}, \quad a=\frac{k}{1+k} \frac{\gamma-1}{\gamma} .
$$

The variable $V$ is closely related to the fluid velocity gradient. Since the term $a \alpha$ and $(a-1) \alpha$ in the brackets are merely corrections to constant coefficients of $0(1)$, the properties of Eq. (2.4) would not be altered if we had neglected these terms in order to simplify further algebra. Thus the approximate differential equation

$$
\alpha w^{2} V \frac{d V}{d w}+V\left(1-w^{2}\right)-w\left(1-\beta w^{2}\right)=0
$$

in the phase space $(w, V)$ is expected to exhibit all important features of the original system, Eqs. $(1.9-1.12)$, for $\mu^{*}=1$. An equation similar to (2.6) was derived by Sakurai [4] and later was discussed in detail by Levey [5] for source flow in a real fluid.

2.2. Properties of the solution curves in phase space. Equation (2.6) is nonlinear and cannot be integrated. However, several important features of the solutions can be readily seen by studying the properties of the vector field $(w, V)$ defined by Eq. (2.6), such as the type of its singular points, the curves of zero slope and zero curvature together with some obvious isoclines.

2.2a. The curve of zero slope; the inviscid solution. Let $G_{1}$ be the curve on which $d V / d w$ given in Eq. (2.6) vanishes, $G_{1}$ is then given by

$$
V=w\left(1-\beta w^{2}\right)\left(1-w^{2}\right)^{-1},
$$

which is also the inviscid solution in $w-V$ plane. The function $V_{1}(w)$ given by Eq. (2.7) has a simple pole at $w=1$ (the fictitious sonic circle), and two zeros at $w=0$ and $w=\beta^{-1 / 2}$ which correspond respectively to the subsonic and supersonic branch at $r=\infty$. Near the origin, $V_{1}(w)$ given by Eq. (2.7) has the following power series expansion

$$
V_{1}=w+(1-\beta) w^{3}+(1-\beta) w^{5}+(1-\beta) w^{7}+\cdots,
$$

which starts from $w=0$ with slope unity. Near the point $w=\beta^{-1 / 2}$, the expansion of $V_{1}(w)$ is

$$
\begin{aligned}
V_{1}=\frac{2 \beta x}{1-\beta}\left[1-\frac{1+3 \beta}{2(1-\beta)}\left(\beta^{1 / 2} x\right)+\frac{1+6 \beta+\beta^{2}}{2(1-\beta)^{2}}\left(\beta^{1 / 2} x\right)^{2}\right. \\
\\
\left.\quad-\frac{1+10 \beta+5 \beta^{2}}{2(1-\beta)^{3}}\left(\beta^{1 / 2} x\right)^{3}+\cdots\right],
\end{aligned}
$$

where

$$
x=w-\beta^{-1 / 2} \text {. }
$$

This branch of the curve $G_{1}$ starts from $\left(\beta^{-1 / 2}, 0\right)$ with the slope $2 \beta /(1-\beta)$. The curve $G_{1}$ divides the infinite strip $0 \leq w \leq \beta^{-1 / 2}$ into regions of positive and negative slope as shown in Fig. 2. In this strip, $d V / d w$ given in Eq. (2.6) becomes infinite only on (i) $V=0,0<w<\beta^{-1 / 2}$, and (ii) $w=0, V \neq 0$. Besides, $w=\beta^{-1 / 2}, V \neq 0$ is also an isocline on which

$$
d V / d w=(1-\beta) / \alpha
$$




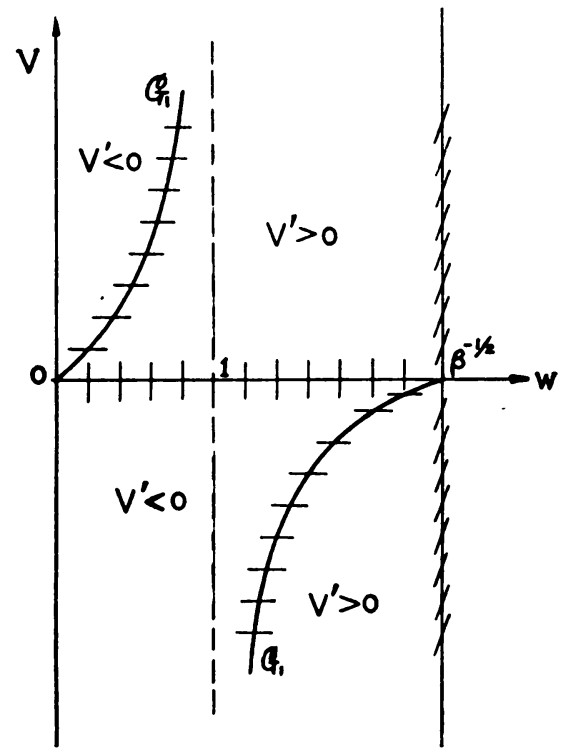

FIG. 2. Regions of positive and negative slopes; isoclines.

2.2b. Properties of the singular points. The only singular points of Eq. $(2.6)$ are $(0,0)$ and $\left(\beta^{-1 / 2}, 0\right)$. The origin is a singular point of higher order. But if one sketches the vector field $(d w / d t, d V / d t)$ defined by

$$
d w / d t=\alpha w^{2} V, \quad d V / d t=w\left(1-\beta w^{2}\right)-V\left(1-w^{2}\right)
$$

along a simple closed curve in the neighborhood of the origin with the origin in its interior, one finds that the Poincaré index (see [8], p. 45) of this singularity is equal to -1 . Thus the origin is a saddle point through which only two solution curves may pass. One of these is $w=0$ which either yields a trivial solution $(V=0)$ or has no physical meaning $(V \neq 0)$. The other solution curve starts from the origin with slope equal to 1 (which coincides there with the inviscid solution) and thus represents the only possible radial sink flow with stagnation at $r=\infty$. By substitution of a power series into Eq. (2.6) (or by ordinary iteration), the asymptotic value, for small $\alpha$, of this solution near the origin is found to be

$$
\begin{aligned}
V \cong w & +[(1-\beta)-\alpha] w^{3}+\left[(1-\beta)-\alpha(5-4 \beta)+4 \alpha^{2}\right] w^{5} \\
& +\left[(1-\beta)-\alpha\left(14-16 \beta+3 \beta^{2}\right)+10 \alpha^{2}(4-3 \beta)-27 \alpha^{3}\right] w^{7}+\cdots .
\end{aligned}
$$

The point $\left(\beta^{-1 / 2}, 0\right)$ is a singular point of regular type. In the neighborhood of $x \equiv w-\beta^{-1 / 2}=0, V=0$, Eq. (2.6) becomes

$$
\frac{d V}{d x}=\frac{-2 \beta x+(1-\beta) V+P(x, V)}{\alpha V+Q(x, V)}
$$

in which $P$ and $Q$ vanish like $x^{2}+V^{2}$ as $x, V \rightarrow 0$. Thus the singularity [Ref. $8 \mathrm{pp}$. 37-44] is (i) a nodal point if $\alpha \leq(1-\beta)^{2} /(8 \beta)$, (for air, $\beta=1 / 6, \alpha \leq 25 / 48$ ), or (ii) a spiral point if $\alpha>(1-\beta)^{2} /(8 \beta)$. As the problem will be confined to the case $\alpha \ll 1$, 
we shall only consider this singularity to be a nodal point (which changes to a saddle point for source flow [5]). All solution curves passing through this point will have at this point two distinct slopes which can be calculated from the secular equation of Eq. (2.12), namely,

$$
\left|\begin{array}{cc}
\lambda & -\alpha \\
2 \beta & \lambda-(1-\beta)
\end{array}\right|=0,
$$

which has two unequal positive roots

$$
\lambda_{1}=(1-\beta)-2 \alpha \beta /(1-\beta)+0\left(\alpha^{2}\right), \quad \lambda_{2}=2 \alpha \beta /(1-\beta)+0\left(\alpha^{2}\right) .
$$

From these two eigen-values two eigen-vectors associated with Eq. (2.12) at $(x=0$, $V=0$ ) can be obtained. By using this result it can be shown that the solution curves passing through $(x=0, V=0)$ have near this point the following parametric representation

$$
V-\frac{2 \beta}{1-\beta} x=C_{1} e^{\lambda_{1} t}, \quad V-\left(\frac{1-\beta}{\alpha}-\frac{2 \beta}{1-\beta}\right) x=C_{2} e^{\lambda_{2} t} .
$$

Since $\lambda_{1}>\lambda_{2}>0, V, x \rightarrow 0$ as $t \rightarrow-\infty$. It also follows from the above equations that there is an infinite number of solution curves, corresponding to arbitrary $C_{1}$ and $C_{2}$ $\left(C_{2} \neq 0\right)$ which have the asymptotic value

$$
V \cong \frac{2 \beta}{1-\beta} x+C|x|^{(1-\beta)^{2 /(2 \alpha \beta)}} \quad \text { near }(x=0, V=0)
$$

and, in addition, there is another solution curve $\left(\sim C_{2}=0\right)$ passing through this point, of the value

$$
V \cong\left(\frac{1-\beta}{\alpha}-\frac{2 \beta}{1-\beta}\right) x \quad \text { near }(x=0, V=0) .
$$

The first group of solutions, given by Eq. (2.15a), have the same limiting value at $r=\infty$ as the inviscid solution and hence represent the many possible sink flows starting with maximum velocity $w=\beta^{-1 / 2}$ at $r=\infty$, while the solution given by Eq. (2.15b) is physically irrelevant. The asymptotic value, for small $\alpha$, of this physically significant solution near the point $x \equiv w-\beta^{-1 / 2}=0$ is

$$
\begin{aligned}
V \cong & \frac{2 \beta x}{1-\beta}\left\{\left[1+\frac{2 \alpha \beta}{(1-\beta)^{2}}+\frac{8 \alpha^{2} \beta^{2}}{(1-\beta)^{4}}+0\left(\alpha^{3}\right)\right]\right. \\
& -\frac{\beta^{1 / 2} x}{2(1-\beta)}\left[(1+3 \beta)+2 \alpha \frac{\beta(3+13 \beta)}{(1-\beta)^{2}}+0\left(\alpha^{2}\right)\right] \\
& \left.+\frac{\beta x^{2}}{2(1-\beta)^{2}}\left[\left(1+6 \beta+\beta^{2}\right)+2\left(5+42 \beta+33 \beta^{2}\right) \frac{\alpha \beta}{(1-\beta)^{2}}+0\left(\alpha^{2}\right)\right]+\cdots\right\} \\
& +C|x|^{(1-\beta)^{2 / 2 \alpha \beta}},
\end{aligned}
$$

where $C$ is an arbitrary constant. The last term follows from Eq. (2.15a). 
2.2c. The curve of zero curvature. The second derivative of the solution $V(w)$ can be deduced from (2.6). Let $G_{2}$ be the curve on which $d^{2} V / d w^{2}$ vanishes, the equation for $G_{2}$ is, except where $w V$ is zero, given by:

$$
2 V^{3}-w\left(1+\beta w^{2}\right) V^{2}+\alpha^{-1}\left(1-\beta w^{2}\right)\left[V\left(1-w^{2}\right)-w\left(1-\beta w^{2}\right)\right]=0 .
$$

The function $V_{2}(w)$ satisfying this equation has the following properties:

(i) For small values of $\alpha, V_{2}$ has only one real value for either

$$
0 \leq w<1+\frac{3}{2}[(1-\beta) \alpha / 2]^{1 / 3} \quad \text { or } \quad w>\beta^{-1 / 2}\left[1+\frac{\alpha}{4(1-\beta)}\right]
$$

$V_{2}$ has three real values for

$$
1+\frac{3}{2}[(1-\beta) \alpha / 2]^{1 / 3} \leq w \leq \beta^{-1 / 2}\left[1+\frac{\alpha}{4(1-\beta)}\right] ;
$$

$V_{\mathfrak{z}}$ has two equal real values at

$$
w=1+\frac{3}{2}[(1-\beta) \alpha / 2]^{1 / 3} ; \quad w=\beta^{-1 / 2}\left[1+\frac{\alpha}{4(1-\beta)}\right] \quad \text { and } \quad w=\beta^{-1 / 2} \text {. }
$$

(ii) The curve $G_{2}$ starts from the origin with slope equal to unity and has, in the neighborhood of the origin, the following expansion:

$V_{2} \cong w+[(1-\beta)-\alpha] w^{3}+\left[(1-\beta)-\alpha(5-4 \beta)+4 \alpha^{2}\right] w^{5}$

$$
+\left[(1-\beta)-\alpha\left(14-16 \beta+3 \beta^{2}\right)+2 \alpha^{2}(17-12 \beta)-21 \alpha^{3}\right] w^{7}+\cdots .
$$

(iii) The curve $G_{2}$ crosses $w=1$ at

$$
V_{2}(1)=(1-\beta)^{2 / 3}(2 \alpha)^{-1 / 3}\left[1+0\left(\alpha^{1 / 3}\right)\right]
$$

with the slope

$$
d V_{2} / d w=(2 / 3)(1-\beta)^{1 / 3}(2 \alpha)^{-2 / 3}\left[1+0\left(\alpha^{1 / 3}\right)\right] .
$$

(iv) When $w=\beta^{-1 / 2}, V_{2}^{2}=0$, and $V_{2}=\beta^{-1 / 2}$. Thus $G_{2}$ has a double point at $\left(\beta^{-1 / 2}, 0\right)$, where the curve has two different slopes $2 \beta(1-\beta)^{-1}[1+0(\alpha)]$ and $(1-\beta) \alpha^{-1}[1+0(\alpha)]$. Near this point these two branches of the curve have the following expansions:

$$
\begin{aligned}
V_{2}^{(1)} \cong \frac{2 \beta x}{(1-\beta)}\left\{\left[1+\frac{2 \alpha \beta}{(1-\beta)^{2}}+\frac{8 \alpha^{2} \beta^{2}}{(1-\beta)^{4}}+0\left(\alpha^{3}\right)\right]\right. \\
\quad-\frac{\beta^{1 / 2} x}{2(1-\beta)}\left[(1+3 \beta)+4 \alpha \frac{\beta(7 \beta-1)}{(1-\beta)^{2}}+0\left(\alpha^{2}\right)\right] \\
\left.\quad+\frac{\beta x^{2}}{2(1-\beta)^{2}}\left[\left(1+6 \beta+\beta^{2}\right)+0(\alpha)\right]+\cdots\right\}
\end{aligned}
$$

and

$$
V_{2}^{(2)} \cong \frac{1-\beta}{\alpha} x\left[1-\frac{2 \alpha \beta}{(1-\beta)^{2}}+0\left(\alpha^{2}\right)\right]+\cdots
$$


(v) The slope of the curve $G_{2}$ becomes infinite at

and

$$
w \sim 1+\frac{3}{2}[(1-\beta) \alpha / 2]^{1 / 3}, \quad V \sim-\alpha^{-1 / 3}[(1-\beta) / 2]^{2 / 3}
$$

$$
w \sim \beta^{-1 / 2}\left[1+\frac{\alpha}{4(1-\beta)}\right], \quad V \sim \frac{1}{2} \beta^{-1 / 2} .
$$

(vi) For $w \gg 1, V_{1} \sim \beta w, V_{2} \sim \beta w^{3} / 2$.

The curve $G_{2}$ divides the ( $\left.w-V\right)$ plane into regions of positive and negative curvatures as shown in Fig. 3.

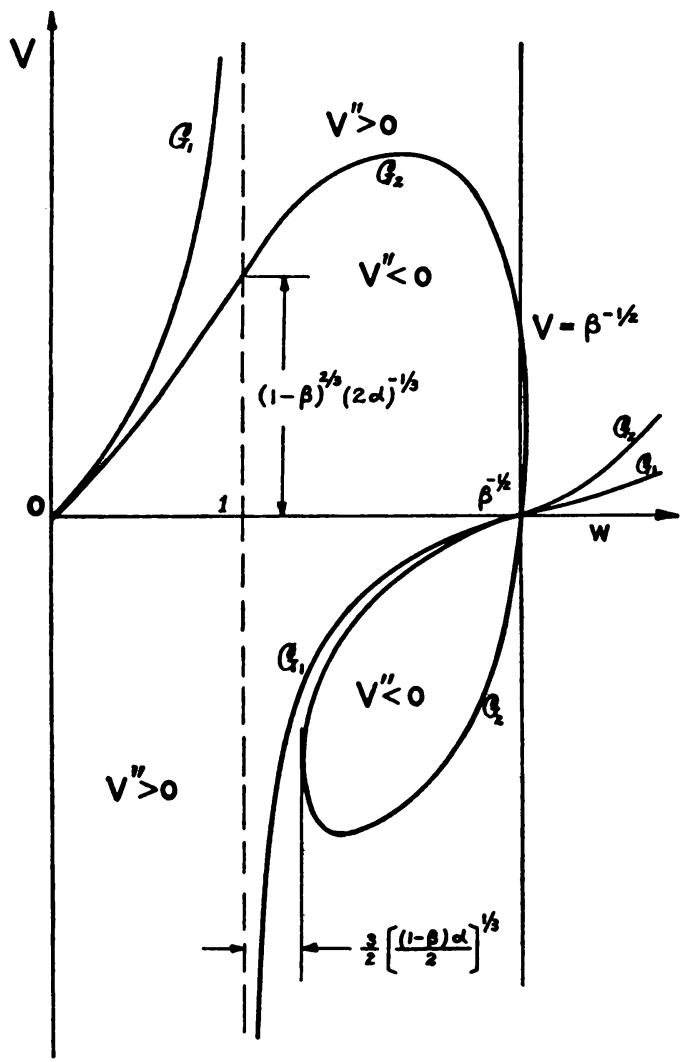

Fig. 3. Regions of positive and negative curvature.

2.2d. Sketch of solution curves $V(w)$; definition of cylindrical shock. The above discussions on isoclines, types of singularities, regions of positive and negative slope and curvature, enable the solution curves to be sketched.

First let us consider the solution curve starting from the origin. Comparison of Eqs. (2.8), (2.11) and (2.18) shows that, for $\alpha$ and $w$ both small,

$$
V_{1}(w)>V(w)>V_{2}(w),
$$

the difference $\left(V_{1}-V\right)$ being of $0\left(\alpha w^{3}\right)$ while $\left(V-V_{2}\right) \sim 0\left(\alpha^{2} w^{7}\right)$. Thus the solution curve $V(w)$ lies in between $G_{1}$ and $G_{2}$ where the slope and curvature of $V(w)$ are both 
positive. A careful study of the slope of $V(w)$ and $G_{2}$ indicates that the solution curve lies above $G_{2}$ for $w>0$. Hence $V(w)$ is a monotonic increasing function of $w$ with increasing slope, passing through $w=1$ between the points $V=(1-\beta) / \alpha$ and $V=$ $(1-\beta)^{2 / 3}(2 \alpha)^{-1 / 3}$, and finally ending up at $w=\beta^{-1 / 2}$ with the slope $d V / d w=(1-\beta) / \alpha$ (cf. Eq. 2.10 and Fig. 4).

As previously shown in Eq. (2.16), there is an infinite number of solution curves starting from $\left(w=\beta^{-1 / 2}, V=0\right)$ with the same slope $2 \beta /(1-\beta)$. However, for $\left(w-\beta^{-1 / 2}\right)$ and $\alpha$ both small enough, comparison of Eqs. (2.9), (2.16) and (2.20) again shows that all these solutions satisfy, for small negative $\left(w-\beta^{-1 / 2}\right)$, the following inequality

$$
V(w)<V_{2}^{(1)}(w)<V_{1}(w),
$$

as shown in Fig. 4. As $w$ decreases from $\beta^{-1 / 2}, V$ (for every finite $C$ in Eq. 2.16) decreases with increasing slope until it intercepts $G_{2}$ with a positive slope. For further decrease

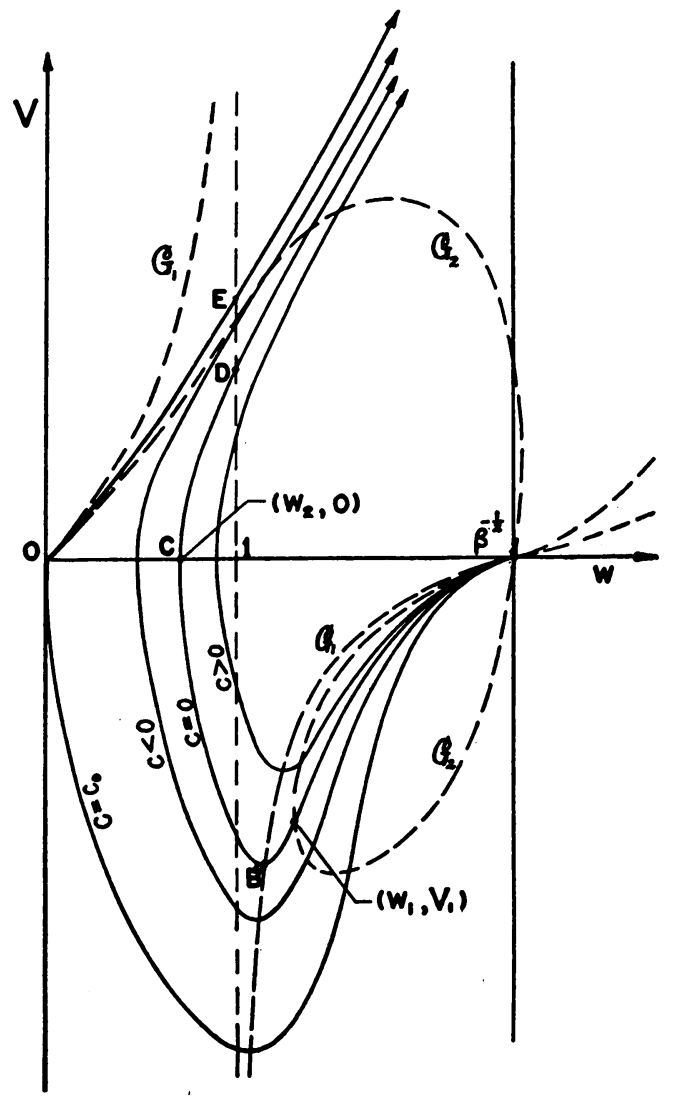

FIG. 4. Sketch of the solution curves in the phase space.

in $w$, the curve $V$ should lie above a straight line with this positive slope at the point of intersection because $V$ has positive curvature in this region. Hence the solution curves will eventually meet $G_{1}$ with zero slope. From there on, for further decrease in $w, V$ increases from negative values and later crosses $V=0$ with infinite slope at some point 
in between $w=0$ and $w=\beta^{-1 / 2}$, as can be shown by the method of bounding curves, and will be made explicit in our later calculation. Further extension of these solution curves shows that $V$ increases with increasing $w$ and finally approaches asymptotically to the subsonic branch solution which starts from the origin. There is a particular value of the integration constant $C$ (in Eq. 2.16), say, $C=C_{0}<0$, for which the solution curve finally ends up at the origin with infinite slope. For $C<C_{0}$, the solution ceases to have physical meaning. On the other hand, the solution curves for $C>C_{0}$ have a very interesting feature that these viscous solutions all exhibit the transition process from the inviscid supersonic branch toward the viscous subsonic branch. Let us consider, in particular, the solution with $C=0$. It first intercepts $G_{2}$ at $\left(w_{1}, V_{1}\right)$, say, and then crosses $V=0$ at $w=w_{2}$. Since $w_{1}>1$ and $w_{2}<1$ (as will be shown later), the flow between these two states may thus be defined as that of a "cylindrical shock".* Inspired by the result obtained in Eqs. $(2.19 \mathrm{a}, \mathrm{b})$, we see that the equation governing such a cylindrical shock flow can be approximated by the following similarity transformation

$$
w=1+\alpha^{1 / 3} \bar{w}, \quad V=\alpha^{-1 / 3} \bar{v},
$$

which will render all terms in Eq. (2.6) equally important in this flow region, that is, for vanishing $\alpha$,

$$
\bar{v} d \bar{v} / d \bar{w}=2 \bar{w} \bar{v}+(1-\beta) .
$$

Since this equation governs the flow of both branches near the sonic speed, it may be called the "equation for cylindrical transonic flow".

2.3. Sketch of solution curves $w(\eta)$ in physical space. From the definition of $V(w)$ given by Eq. (2.3), we have

$$
\eta=-\int^{w}[V(w)]^{-1} d w+h
$$

where the integral stands for an indefinite integral and

$$
h=\frac{1}{\gamma-1} \log \left(\frac{2}{\gamma+1}\right)
$$

so that $\eta$ tends to its inviscid solution for $\eta$ large.

Now for the subsonic branch, $V(w) \geq 0$, hence $\eta$ is a monotonically decreasing function of $w$. Moreover, for some value of $w, V(w)$ is less than its corresponding inviscid solution, $V_{1}(w)$ (see Eq. 2.22). Hence, from Eq. (2.26),

$$
\eta_{\mathrm{vis}}(w)<\eta_{\mathrm{invie}}(w) \text {. }
$$

In other words, at every $\eta,(w)_{v i s}$ is slowed down from its inviscid value due to the viscous effect.

*This terminology is adopted by both Sakurai [4] and Levey [5] to describe such type of flow. The term "shock" is borrowed from its conventional meaning to indicate the transition from one branch to the other, though the transition is rather different from that occurring in a plane normal shock. Perhaps this terminology relates more closely to the conventional meaning of a shock for the constant $C$ slightly greater than $C_{0}$ (see Fig. 4), because then the jump in $w$ and the slope $d w / d \eta$ in transition become greater, and the position of transition is farther out from $r=r_{1}$ (see Fig. 5). But since there is no adequate criterion to distinguish one from another value of $C$, we shall retain this name. Another terminology, the dissipation layer, is suggested by Prof. H. S. Tsien to avoid this ambiguity and, in addition, to stress the importance of viscous effects in this layer. 
For the supersonic branch starting from $w=\beta^{-1 / 2}, V(w) \leq 0$ for $w_{2} \leq w \leq \beta^{-1 / 2}$, hence in this interval $\eta$ is a monotonically increasing function of $w$. At $w=w_{2},(\sim \eta=$ $\eta_{2}$, say), $(d w / d \eta)=-V\left(w_{2}\right)=0$, and, from Eq. (2.6), $\left(d^{2} w / d \eta^{2}\right)=(V d V / d w)_{0-0}=$ $\left(1-\beta w_{2}\right) /\left(\alpha w_{2}\right)>0$, therefore $w\left(\eta_{2}\right)=w_{2}$ is the only minimum of $w$ on this branch. For $\eta \leq \eta_{2}, \eta$ decreases with increasing $w$. Furthermore, because of the relation given in Eq. (2.23), we have

$$
\eta_{\text {vio }}(w)>\eta_{\text {invis }}(w) \quad \text { for } \quad w>1, V \leq 0 .
$$

The above properties of the solution enable the solution curves to be sketched, as shown in Fig. 5. As the constant $C$ decreases from zero, the minimum value decreases and the jump in $w$ increases while the jump takes place farther upstream.

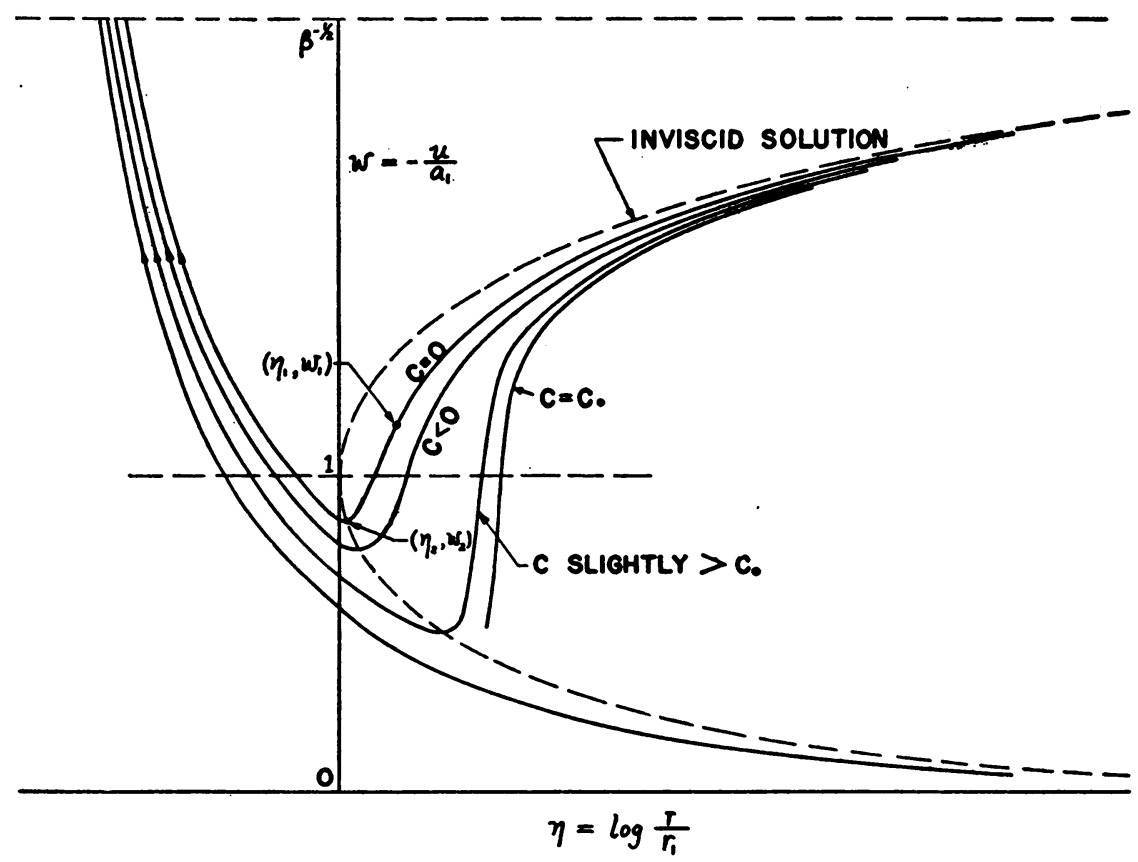

Fig. 5. Sketch of the solution curves in the physical space.

It follows from Eq. (2.24) that if we introduce

$$
\eta=\alpha^{2 / 3} \xi, \quad w=1+\alpha^{1 / 3} \bar{w},
$$

then Eq. (2.25) becomes

$$
\frac{d^{2} \bar{w}}{d \xi^{2}}=-2 \bar{w} \frac{d \bar{w}}{d \xi}+(1-\beta),
$$

which is the equation governing the flow near $w=1, \eta=0$. Integrating this equation once, we obtain

$$
d \bar{w} / d \xi+\bar{w}^{2}=(1-\beta) \xi+\text { const. }
$$

This equation will be integrated and discussed in our final calculation. 
3. Calculation of the solutions by using $P L K$-method*. In this section we shall calculate $w(\eta), \theta(\eta)$ governed by the original system of Eqs. (1.9-1.12). Throughout this section $\mu$ will again be assumed constant so that $\mu^{*}=1$, but no restriction will be imposed on $\sigma$ and $k$. Consequently Eqs. (1.9) and (1.12) become

$$
\begin{gathered}
w^{2} \frac{d w}{d \eta}+\frac{1}{\gamma}\left[w \frac{d \theta}{d \eta}-\theta \frac{d w}{d \eta}-\theta w\right]=-\frac{\gamma+1}{2 \gamma} \alpha\left(\frac{d^{2} w}{d \eta^{2}}-w\right) w^{2}, \\
\theta+\frac{\gamma-1}{2}(1+b \alpha) w^{2}+\frac{\gamma+1}{4 \gamma} \alpha\left[\frac{\sigma^{-1}}{1+k} \frac{d \theta}{d \eta}+(\gamma-1) \frac{d w^{2}}{d \eta}\right]=\frac{\gamma+1}{2},
\end{gathered}
$$

where $\alpha$ is given by Eq. (2.5) and

$$
b=a / \beta=(1+1 / \gamma)(1+1 / k)^{-1} \text {. }
$$

Using the PLK-method, as described below, the generalization to the case $\mu=\mu(T)$ presents no particular difficulty. The calculation for the case when $\mu$ is proportional to local temperature is carried out in Ref. 1 , Sec. 7. The result shows that all important features of flow quantities for $\mu^{*}=1$ remain up to the term of $0\left(\alpha^{2 / 3}\right)$.

It was seen before that, even for a simplified version of these equations, such as Eq. (2.6), the conventional perturbation method merely leads to asymptotic solutions for small $\alpha$ near $w=0$ or $w=\beta^{-1 / 2}$ (see Eqs. 2.11, 2.16) because the coefficient of $w^{n}$ does not diminish as $n \rightarrow \infty$. This asymptotic result fails to be a good approximation to the required solution as $w$ deviates further from $w=0$ or $w=\beta^{-1 / 2}$ and becomes almost useless for calculations near $w=1$. Now let us resort to the PKL-method, which is in essence to expand the solution in terms of power series in $\alpha$ with coefficients as undetermined functions of a parameter $\xi$.

$$
\begin{aligned}
w(\xi) & =\xi+\alpha w^{(1)}(\xi)+\alpha^{2} w^{(2)}(\xi)+\cdots, \\
\eta(\xi) & =\eta^{(0)}(\xi)+\alpha \eta^{(1)}(\xi)+\alpha^{2} \eta^{(2)}(\xi)+\cdots, \\
\theta(\xi) & =\theta^{(0)}(\xi)+\alpha \theta^{(1)}(\xi)+\alpha^{2} \theta^{(2)}(\xi)+\cdots .
\end{aligned}
$$

The need of a parameter $\xi$ to represent the solution and that $w$ starts with the term $\xi$ are clearly suggested by our previous discussions. Substituting these expansions into Eqs. (3.1) and (3.2), noting that

$$
d w / d \eta=w^{\prime} / \eta^{\prime}, \quad d \theta / d \eta=\theta^{\prime} / \eta^{\prime}, \quad d^{2} w / d \eta^{2}=w^{\prime \prime}\left(\eta^{\prime}\right)^{-2}-w^{\prime} \eta^{\prime \prime}\left(\eta^{\prime}\right)^{-3},
$$

where prime stands for $d / d \xi$, and then equating equal powers of $\alpha$, we obtain the zeroth order equations as follows:

$$
\begin{gathered}
{\left[\gamma \xi^{2}+\xi^{2} \frac{d}{d \xi}\left(\theta^{(0)} / \xi\right)-\xi \theta^{(0)} \eta^{(0)^{\prime}}\right]\left(\eta^{(0)^{\prime}}\right)^{2}=0,} \\
{\left[\theta^{(0)}-\left(\frac{\gamma+1}{2}-\frac{\gamma-1}{2} \xi^{2}\right)\right] \eta^{(0)^{\prime}}=0 .}
\end{gathered}
$$

If we choose $\eta^{(0) \prime}$ different from zero, then we have the zeroth order solution

$$
\begin{gathered}
\theta^{(0)}(\xi)=\frac{\gamma+1}{2}-\frac{\gamma-1}{2} \xi^{2}, \\
\eta^{(0)}(\xi)=-\log \xi-\frac{1}{\gamma-1} \log \left|\frac{\gamma+1}{2}-\frac{\gamma-1}{2} \xi^{2}\right| .
\end{gathered}
$$

*See the footnote in the Introduction. 
The integration constant in Eq. (3.7b) has been so chosen that when $\alpha=0, \xi \equiv w$ and $\eta^{(0)}(w)$ agrees with inviscid solution.

The coefficient of $\alpha$ in the expanded equations gives the following first order equations:

$$
\begin{gathered}
\theta^{(1)}+(\gamma-1) \xi w^{(1)}=-\frac{\gamma-1}{2} b \xi^{2}+\frac{\gamma+1}{2} \epsilon \xi^{2}\left(1-\beta \xi^{2}\right)\left(1-\xi^{2}\right)^{-1}, \\
\left(\eta^{(0)^{\prime}}\right)^{2}\left\{\left(2 \gamma \xi+\theta^{(0)}\right) w^{(1)}+\left(\gamma \xi^{2}-\theta^{(0)}\right) w^{(1)^{\prime}}+\xi^{2} \frac{d}{d \xi}\left(\theta^{(1)} / \xi\right)-\xi \theta^{(0)} \eta^{(1) \prime}\right. \\
\left.-\left(\xi \theta^{(1)}+w^{(1)} \theta^{(0)}\right) \eta^{(0)}\right\}=\frac{\gamma+1}{2} \xi^{2}\left[\eta^{(0) \prime}+\xi\left(\eta^{(0) \prime}\right)^{3}\right],
\end{gathered}
$$

where $b$ is given by Eq. (3.3) and

$$
\epsilon=\frac{\gamma-1}{\gamma}\left[1-\frac{1}{2 \sigma(1+k)}\right] \text {. }
$$

Substituting Eqs. (3.7) and (3.8a) into (3.8b), and rearranging the terms, we finally obtain [see Ref. 1, p. 25]:

$\frac{d}{d \xi}\left[\eta^{(0)} w^{(1)}-\eta^{(1)}\right]$

where

$$
=(1-\epsilon)\left[\frac{2 \xi}{\left(1-\xi^{2}\right)^{2}}+\frac{\gamma \xi}{\xi^{2}-1}\right]+A \frac{2 \beta \xi}{\left(\beta \xi^{2}-1\right)^{2}}+B \frac{2 \beta \xi}{\left(\beta \xi^{2}-1\right)},
$$

$$
A=\frac{1}{2 \beta^{2}}(1-a)(1-\beta), \quad B=\frac{1}{2 \beta}\left[\frac{1-a}{\beta}-(a-\epsilon)-\frac{(1+\epsilon) 2 \beta}{1-\beta}\right]
$$

and the constants $a, b, \epsilon$ are given by Eqs. (3.3) and (3.8c). Now in order to ascribe to $w^{(1)}$ a possibly lowest order singularity at $\xi=1$ to improve the convergence of the series, we decompose Eq. (3.9) as follows:

$$
\begin{gathered}
\frac{d}{d \xi}\left[\eta^{(0) '} w^{(1)}\right]=A \frac{2 \beta \xi}{\left(\beta \xi^{2}-1\right)^{2}}+B \frac{2 \beta \xi}{\left(\beta \xi^{2}-1\right)}, \\
\frac{d}{d \xi} \eta^{(1)}=-(1-\epsilon)\left[2 \xi\left(\xi^{2}-1\right)^{-2}+\gamma \xi\left(\xi^{2}-1\right)^{-1}\right] .
\end{gathered}
$$

The solution of Eqs. (3.10a, b) is then

$$
\begin{gathered}
w^{(1)}(\xi)=-A \xi\left(1-\xi^{2}\right)^{-1}-B \xi\left(1-\beta \xi^{2}\right)\left(1-\xi^{2}\right)^{-1} \log \left|\frac{\gamma+1}{2}-\frac{\gamma-1}{2} \xi^{2}\right|, \\
\eta^{(1)}(\xi)=-(1-\epsilon)\left[\left(1-\xi^{2}\right)^{-1}+\frac{\gamma}{2} \log \left|1-\xi^{2}\right|\right] .
\end{gathered}
$$

The integration constant of Eq. (3.9) is first absorbed in $\eta^{(1)}$ and is then omitted because of its negligible contribution near $\xi=1$. In the above first order solution, $w^{(1)}$ and $\eta^{(1)}$ have singularities at $\xi=1$ of the same order. After $w^{(1)}$ is so determined, $\theta^{(1)}(\xi)$ is then given by Eq. (3.8a).

Proceeding in a similar manner to obtain the second order equations by equating terms with $\alpha^{2}$, we find that the resulting equations possess solution of quite lengthy ex- 
pression, in which $\eta^{(2)}(\xi)$ starts with the term $2(1-\beta)(1-\epsilon)\left(1-\xi^{2}\right)^{-4}$ followed by terms of $0\left(\left(1-\xi^{2}\right)^{-3}\right)$, while $w^{(2)}(\xi)$ still can be made to be of $0\left(\left(1-\xi^{2}\right)^{-1}\right)$. Therefore the final solution can be expressed parametrically as follows:

$$
\begin{aligned}
& w(\xi)=\xi-\alpha\left[A \frac{\xi}{1-\xi^{2}}+B \frac{\xi\left(1-\beta \xi^{2}\right)}{1-\xi^{2}} \log \left|\frac{\gamma+1}{2}-\frac{\gamma-1}{2} \xi^{2}\right|\right] \\
& +0\left(\frac{\alpha^{2}}{1-\xi^{2}}\right) \\
& \eta(\xi)=-\left[\log \xi+\frac{1}{\gamma-1} \log \left|\frac{\gamma+1}{2}-\frac{\gamma-1}{2} \xi^{2}\right|\right] \\
& -\alpha(1-\epsilon)\left[\frac{1}{1-\xi^{2}}+\frac{\gamma}{2} \log \left|1-\xi^{2}\right|\right]+\alpha^{2} \frac{2(1-\beta)(1-\epsilon)}{\left(1-\xi^{2}\right)^{4}} \\
& +0\left(\frac{\alpha^{2}}{\left(1-\xi^{2}\right)^{3}}, \frac{\alpha^{3}}{\left(1-\xi^{2}\right)^{7}}\right)+C\left|\frac{\gamma+1}{2}-\frac{\gamma-1}{2} \xi^{2}\right|^{(1-\beta) \cdot /(2 \alpha \beta)},
\end{aligned}
$$

where $A, B$ are constants given in Eqs. (3.9a) and $C$ is an arbitrary constant for the solution starting from $\xi=\beta^{-1 / 2}$, but $C=0$ for the solution starting from $\xi=0$. The term with $C$ is included in the above equation in order to exhibit the behavior of solution curves near the nodal point $\left(w=\beta^{-1 / 2}, \eta=\infty\right)$, as previously discussed in Sec. $2.2 \mathrm{~b}$. This term is of higher order than any finite power of $\alpha$. In fact, it has an essential singularity at $\alpha=0$ and hence cannot be obtained by the expansion procedure. The value of $\theta$ is given by

$$
\begin{aligned}
\theta(\xi)=\left(\frac{\gamma+1}{2}-\frac{\gamma-1}{2} \xi^{2}\right) \\
+\alpha(\gamma-1)\left[A \frac{\xi^{2}}{1-\xi^{2}}+B \frac{\xi^{2}\left(1-\beta \xi^{2}\right)}{1-\xi^{2}} \log \left|\frac{\gamma+1}{2}-\frac{\gamma-1}{2} \xi^{2}\right|\right. \\
\left.\quad-\frac{b}{2} \xi^{2}+\frac{\epsilon}{2 \beta} \frac{\xi^{2}\left(1-\beta \xi^{2}\right)}{1-\xi^{2}}\right]+0\left(\frac{\alpha^{2}}{\left(1-\xi^{2}\right)^{2}}\right) .
\end{aligned}
$$

Several interesting features of the above solution may be mentioned here. (i) $\epsilon=0$ for $\sigma=[2(1+k)]^{-1}(=3 / 4$ if $k=-1 / 3$, see Eq. 3.8c). With the value of $\sigma$ and $k$ lying in the experimental range, $\epsilon$ is still a small number. Consequently, the variation in $\sigma$ and $k$ only contribute a small correction to the coefficients of $0(1)$ in the solution. This fact confirms our previous statement in Sec. 2.1. (ii) By substituting Eq. (3.12) into $\mathrm{Eq}$. (2.2), it can be found that the arbitrary constant $E$ is of $0(\alpha \epsilon)$. (iii) The most important property of the above solution is that it does not provide an approximate solution with uniform accuracy over the interval of $\xi$ such that $0 \leq w \leq \beta^{-1 / 2}$. As $\xi$ approaches unity, the higher order terms, especially in $\eta(\xi)$, become more important relative to the zeroth order term. More precisely, the solution is good only for $0 \leq \xi \leq$ $1-K \alpha^{1 / 3}$ and $1+K \alpha^{1 / 3} \leq \xi \leq \beta^{-1 / 2}, K$ being a positive constant of $0(1)$. At $\xi=1$ $\pm K \alpha^{1 / 3}$, all terms in the expression for $\eta(\xi)$ become of the same order, $0\left(\alpha^{2 / 3}\right)$; but the convergence can be made sufficiently rapid by an appropriate choice of the value $K$.

In the subsequent calculation of the solution through the transonic region, we shall only consider a particular solution with $C=0$ and $\epsilon=0$ in Eqs. (3.12). Furthermore, it 
has been found convenient to take $K=2(\gamma+1)^{-1 / 3}$. With this value of $K$, we obtain, from Eqs. (3.12), the following result:

(i) on supersonic branch, at $w=1+2 \alpha^{1 / 3}(\gamma+1)^{-1 / 3}$,

$$
\eta=2.28(\gamma+1)^{1 / 3} \alpha^{2 / 3} \text { and } \quad d w / d \eta=0.615 \alpha^{-1 / 3}(\gamma+1)^{-2 / 3} ;
$$

(ii) on subsonic branch, at $w=1-2 \alpha^{1 / 3}(\gamma+1)^{-1 / 3}$,

$$
\eta=1.766(\gamma+1)^{1 / 3} \alpha^{2 / 3} \text { and } d w / d \eta=-0.477 \alpha^{-1 / 3}(\gamma+1)^{-2 / 3} .
$$

These values will serve for the boundary conditions imposed on the transonic solution to be obtained below.

That the PLK-method is powerful in solving this problem can still be stressed further by the following argument. As the first order term in the expression for $w(\xi)$ (see 3.12a) is quite unimportant in the aforementioned regions of $\xi$, one perhaps would try, instead of Eq. (3.4), a simpler expansion

$$
\eta(w)=\eta^{(0)}(w)+\alpha \eta^{(1)}(w)+\cdots
$$

and a similar expansion for $\theta$ in terms of $w$. It can be shown that the above expansion will yield a solution in which $\eta^{(0)}$ is identical to inviscid solution, but $\eta^{(1)}$ has, in addition to a simple pole at $w=1$, a pole and a logarithmic singularity at $w=\beta^{-1 / 2}$. Consequently the assumed expansion (3.14) becomes invalid for $r$ large on the supersonic branch, and thus leads to an erroneous result.

4. The solution in the transonic region. To obtain an approximate solution in the transonic region, as discussed in Secs. 2.2d, 2.3 (see Eqs. 2.24, 2.25 and 2.29) and also as guided by the boundary conditions (3.13), first we distort the independent variable by the transformation

$$
\eta=\alpha^{2 / 3} \xi
$$

and then expand $w, \theta$ into the form:

$$
\begin{gathered}
w(\xi)=1+\alpha^{1 / 3} w^{(1)}(\xi)+\alpha^{2 / 3} w^{(2)}(\xi)+\alpha w^{(3)}(\xi)+\cdots, \\
\theta(\xi)=1+\alpha^{1 / 3} \theta^{(1)}(\xi)+\alpha^{2 / 3} \theta^{(2)}(\xi)+\alpha \theta^{(3)}(\xi)+\cdots .
\end{gathered}
$$

With $\epsilon=0(\sim 2 \sigma(1+k)=1)$, Eqs. (3.1) and (3.2) reduce to

$$
\begin{gathered}
{\left[1-(1-a \alpha) w^{2}\right] \frac{d w}{d \eta}+w\left\{1-[\beta+(a-1) \alpha] w^{2}\right\}=\alpha w^{2} \frac{d^{2} w}{d \eta^{2}},} \\
\theta=\frac{\gamma+1}{2}-\frac{\gamma-1}{2}(1+b \alpha) w^{2}+0\left(\alpha^{2}\right) .
\end{gathered}
$$

Substituting (4.1) and (4.2) into (4.4), we obtain the first order equation:

$$
\frac{d^{2} w^{(1)}}{d \xi^{2}}+2 w^{(1)} \frac{d w^{(1)}}{d \xi}=(1-\beta)
$$

and the second order equation:

$$
\frac{d^{2} w^{(2)}}{d \xi^{2}}+2 \frac{d}{d \xi}\left(w^{(1)} w^{(2)}\right)=\frac{d}{d \xi}\left(w^{(1)}\right)^{3}-(1+\beta) w^{(1)} .
$$

The correction due to the terms with constants $a$ and $b$ enters only in $w^{(3)}$ and higher order terms. 
Now Eq. (4.6) can be integrated once to yield:

where

$$
\frac{d y}{d x}+y^{2}=x+x_{1}
$$

$$
y=\left(\frac{\gamma+1}{2}\right)^{1 / 3} w^{(1)}, \quad x=\left(\frac{\gamma+1}{2}\right)^{-1 / 3} \xi,
$$

and the integration constant $x_{1}$ can be determined by using the boundary condition (3.13) to give

$$
\begin{aligned}
& x_{1}=0.033 \text { for the supersonic branch, } \\
& x_{1}=-0.0056 \text { for the subsonic branch. }
\end{aligned}
$$

The nonlinear equation (4.8) (see also Eq. (2.31)) is an approximation to the NavierStokes equation, a case in which all the inertia, pressure and viscous forces are equally important. Now Eq. (4.8) is of Riccati type, which, by using the transformation

$$
y(x)=\frac{1}{v} \frac{d v}{d x},
$$

can be reduced to a second order linear equation:

$$
\frac{d^{2} v}{d x^{2}}-\left(x+x_{1}\right) v=0 .
$$

The solution of this equation for $\left(x+x_{1}\right)>0$ is

$$
v(z)=M z^{1 / 3}\left[I_{-1 / 3}(z)+N I_{1 / 3}(z)\right], \quad z=\frac{2}{3}\left(x+x_{1}\right)^{3 / 2},
$$

where $I(z)$ is the modified Bessel function of the first kind, and $M, N$ are the integration constants. By using Eq. (4.11), the solution of Eq. (4.8) for $\left(x+x_{1}\right)>0$ is then

$$
y(z)=\left(\frac{3 z}{2}\right)^{1 / 3} \frac{I_{2 / 3}(z)+N I_{-2 / 3}(z)}{I_{-1 / 3}(z)+N I_{1 / 3}(z)}, \quad z=\frac{2}{3}\left(x+x_{1}\right)^{3 / 2} .
$$

The constant $N$ can be determined by using again the condition (3.13).

The continuation of Eq. (4.14) into the region $\left(x+x_{1}\right)<0$ is provided by

where

$$
\begin{array}{ll}
z^{1 / 3} I_{1 / 3}(z)=-\zeta^{1 / 3} J_{1 / 3}(\zeta), & z^{1 / 3} I_{-1 / 3}(z)=\zeta^{1 / 3} J_{-1 / 3}(\zeta), \\
z^{2 / 3} I_{2 / 3}(z)=\zeta^{2 / 3} J_{2 / 3}(\zeta), & z^{2 / 3} I_{-2 / 3}(z)=\zeta^{2 / 3} J_{-2 / 3}(\zeta),
\end{array}
$$

$$
\zeta=z e^{-3 \pi i / 2}=\frac{2}{3}\left[-\left(x+x_{1}\right)\right]^{3 / 2} .
$$

Consequently Eq. (4.14) becomes, for $\left(x+x_{1}\right)<0$,

$$
y(\zeta)=\left(\frac{3 \zeta}{2}\right)^{1 / 3} \frac{J_{2 / 3}(\zeta)+N J_{-2 / 3}(\zeta)}{J_{-1 / 3}(\zeta)-N J_{1 / 3}(\zeta)}, \quad \zeta=\frac{2}{3}\left[-\left(x+x_{1}\right)\right]^{3 / 2}
$$

To discuss the above solution, we first note that the inviscid solution in this transonic region, to the first order approximation within the $O\left(\alpha^{2 / 3}\right)$, is

$$
y^{2}=x,
$$

which has two branches for $x>0$ and gives no solution for $x<0$. Now before we determine the value of $N$ for the corresponding viscous solution, we may also note that the 
general solution, given by (4.14) and (4.16), is a semi-transcendental function of $x_{1}$ and the second integration constant $N$. It can be shown, from the property of $I_{0}(z)$ at large $z$, that in Eq. (4.14)

$$
\begin{array}{lllll}
y \rightarrow x^{1 / 2} & \text { as } & x \rightarrow \infty & \text { when } & N>-1, \\
y \rightarrow-x^{1 / 2} & \text { as } & x \rightarrow \infty & \text { when } & N=-1,
\end{array}
$$

and $y$ has a simple pole at a certain finite $z$ for $N<-1$ (which is, of course, of no physical significance). This result shows that the viscous solutions tend to their respective inviscid values for $x$ large in a manner which implies again that $\left(w=\beta^{-1 / 2}, \eta=\infty\right)$ is a nodal point (admitting more than one value of $N)$ while $(w=0, \eta=\infty)$ is a saddle point (admitting only one value of $N$ ). However, for $x+x_{1}<0$, Eq. (4.16) shows obviously that $y(\zeta)$ has an infinite number of isolated simple poles at $\zeta=\zeta_{n}$ where the denominator vanishes. Since the properties of the solution curves in the $(w, V)$ phase space exhibit no such singularities, the solution (4.16), therefore, represents a good approximation to the real flow only for $\zeta$ lying in the interval $0 \leq \zeta \leq \zeta_{1}-\delta<\zeta_{1}$, where $\zeta_{1}$ is the first pole and $\delta$ is a positive number, appropriately chosen such that $y\left(\zeta_{1}-\delta\right)$ is not yet too large to void our approximation (4.2).

Having obtained the first order solution $w^{(1)}(\xi)=2^{1 / 3}(\gamma+1)^{-1 / 3} y$, the second order equation (4.7) can be then integrated to yield

$$
w^{(2)}(\xi)=e^{-2 \varphi(\xi)} \int \psi(\xi) e^{2 \varphi(\xi)} d \xi,
$$

where

$$
\varphi(\xi)=\int w^{(1)}(\xi) d \xi \quad \text { and } \quad \psi(\xi)=\left[w^{(1)}(\xi)\right]^{3}-(1+\beta) \varphi(\xi)+\text { const. }
$$

It is obvious that $w^{(2)}(\xi)$ is bounded wherever $w^{(1)}(\xi)$ is bounded. Consequently, the approximation is good even if we only take the first two terms in (4.2) and (4.3).

In order to obtain some numerical results, we first determine the value $N$ in Eqs. (4.14), (4.16) by using conditions (3.13) to obtain

$$
\begin{aligned}
& N=-0.585 \text { for the supersonic branch, } \\
& N=-1 \quad \text { for the subsonic branch. }
\end{aligned}
$$

With these values of $x_{1}$ and $N$ (see 4.10 and 4.19), the solutions are plotted in Fig. 6 (by using tables, Ref. [8]) from which several interesting results can be deduced as follows:

(i) For the supersonic branch, the transonic solution starts from point $A$ (see Fig. 6) with the coordinates

$$
w_{1}=1+1.6[(\gamma+1) / 2]^{-1 / 3} \alpha^{1 / 3}, \quad \eta_{1} \doteq 2.9[(\gamma+1) / 2]^{1 / 3} \alpha^{2 / 3} .
$$

After the solution curve passes through a point of inflection $G$ and then crosses the line $y=0(w=1)$ at point $B$ (see also Fig. 4) where

$$
\begin{aligned}
\eta_{B} \doteq 1.02[(\gamma+1) / 2]^{1 / 3} \alpha^{2 / 3}\left(\sim V_{B} \doteq-1.05[(\gamma+1) / 2]^{-2 / 3} \alpha^{-1 / 3},\right. \\
\left.(d V / d w)_{B} \doteq-0.95[(\gamma+1) / 2]^{-1 / 3} \alpha^{-2 / 3}\right),
\end{aligned}
$$




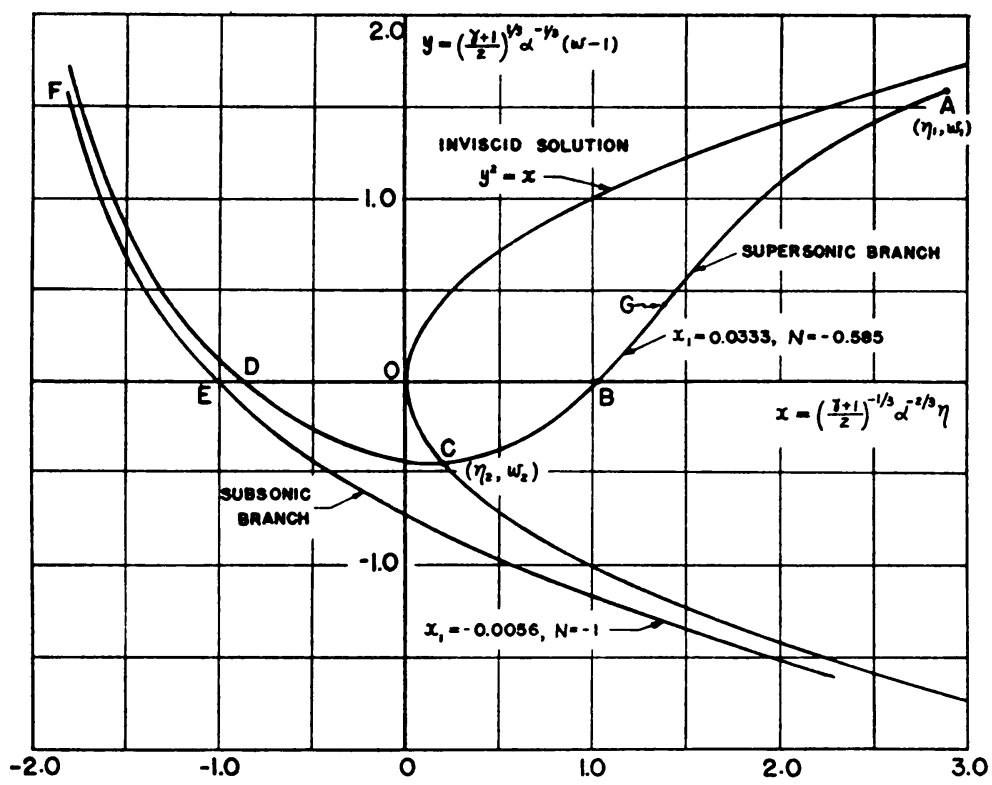

FIG. 6. The flow velocity in the transonic region.

it reaches a minimum when it intercepts the curve $y^{2}=x$ at $C$ where

$$
w_{2} \doteq 1-0.45[(\gamma+1) / 2]^{-1 / 3} \alpha^{1 / 3}, \quad \eta_{2} \doteq 0.2[(\gamma+1) / 2]^{1 / 3} \alpha^{2 / 3} .
$$

It then increases from $w=w_{2}$ to $w=1$ at point $D$ where $\eta_{D} \doteq-0.88\left(\frac{\gamma+1}{2}\right)^{1 / 3} \alpha^{2 / 3}$

$$
\left(\sim V_{D} \doteq 0.91\left(\frac{\gamma+1}{2}\right)^{-2 / 3} \alpha^{-1 / 3}, \quad \frac{d V}{d w} \doteq 1.1\left(\frac{\gamma+1}{2}\right)^{-1 / 3} \alpha^{-2 / 3}\right) .
$$

That is, there is an expansion wave following the cylindrical shock.

(ii) The thickness of the cylindrical shock, as defined in Sec. 2.2d, is

$$
\Delta \eta=\eta_{1}-\eta_{2} \doteq 2.7\left(\frac{\gamma+1}{2}\right)^{1 / 3} \alpha^{2 / 3}, \quad\left(\text { in } r \text {-space, } \Delta r \doteq r_{1} \Delta \eta\right),
$$

across which the velocity has a total variation

$$
\Delta w=w_{1}-w_{2}=2.05[(\gamma+1) / 2]^{-1 / 3} \alpha^{1 / 3} ;
$$

moreover,

$$
w_{1} w_{2}=1+1.15[(\gamma+1) / 2]^{-1 / 3} \alpha^{1 / 3} .
$$

Combining Eqs. (4.22) and (4.23), we obtain

$$
(\Delta w)(\Delta \eta)=5.5 \alpha \text {. }
$$

Comparing these results with those of a plane normal shock (e.g. Ref. [10]), we note first that the plane shock strength $(\sim \Delta w)$ is quite arbitrary while for a cylindrical shock, 
$\Delta w \sim 0\left(\alpha^{1 / 3}\right)$. The expression for shock thickness (Eq. 4.22) shows that $\Delta \eta \sim 0\left(\alpha^{2 / 3}\right)$, although, after combining with $\Delta w$, the expression (4.25) agrees with that of a plane shock [10] within the order of magnitude. The result (4.25) differs, however, from Levey's result for the diffuse shock in a source flow (Ref. [5], Eq. 4.9), in which he explains the discrepancy as due to some degree of choice of the definition of the shock thickness. Our result also indicates that the maximum velocity gradient inside a cylindrical shock is of order $\alpha^{-1 / 3}$, (in contrast to Levey's result: $0\left(\alpha^{-1}\right)$ ), while for a plane shock, the maximum gradient is of order $(\Delta w)^{2} \alpha^{-1}[10]$, which reduces to $0\left(\alpha^{-1 / 3}\right)$ if $\Delta w \sim 0\left(\alpha^{1 / 3}\right)$. The expression (4.24) differs from the Prandtl relation of a plane shock by a term of $0\left(\alpha^{1 / 3}\right)$ which here agrees with Levey's result (Ref. [5], Eq. 4.13). The differences between the present results of cylindrical flow and those of one dimensional plane shock can perhaps be realized by visualizing that the viscous forces exerting on the surfaces $r d \theta$ and $d r$ of a fluid element are indeed of quite different nature from those exerting in plane shock flow, since in the former case, the normal stress acting on the surface $d r$ will have a component in the radial direction.

(iii) On the subsonic branch, $w$ is a monotonically decreasing function of $\eta \cdot w=1$ at point $E$ (see Figs. 6 and 4) where $\eta_{E} \doteq-[(\gamma+1) / 2]^{1 / 3} \alpha^{2 / 3}$ and the velocity gradient $(d w / d \eta)_{E}=-V_{E}=-[(\gamma+1) / 2]^{-2 / 3} \alpha^{-1 / 3}$ (see also Eq. 2.19a), which shows that the solution curve of the subsonic branch passes $w=1$ (see Fig. 4) slightly above the curve $G_{2}$.

(iv) The thermodynamic variables in this flow region can be deduced from Eqs. (4.5), (1.7) and (1.8). That is, in the expansion (4.3) and

$$
\begin{aligned}
& p^{*}(\xi)=1+\alpha^{1 / 3} p^{(1)}(\xi)+\alpha^{2 / 3} p^{(2)}(\xi)+\cdots, \\
& \rho^{*}(\xi)=1+\alpha^{1 / 3} \rho^{(1)}(\xi)+\alpha^{2 / 3} \rho^{(2)}(\xi)+\cdots,
\end{aligned}
$$

we have

$$
\begin{aligned}
\theta^{(1)}(\xi) & =-(\gamma-1)\left(\frac{\gamma+1}{2}\right)^{-1 / 3} y(\xi), \\
p^{(1)} & =\gamma \rho^{(1)}=\frac{\gamma}{\gamma-1} \theta^{(1)},
\end{aligned}
$$

where $y(\xi)$ is given in Eqs. (4.14) and (4.16). The value of $\rho^{(1)}$ and $\theta^{(1)} v s . \eta$ is plotted in Fig. 7. The supersonic branch starts with compression and is then followed by an expansion wave, while the subsonic branch expands continuously. Equation (4.28) simply states that $p^{*}, \rho^{*}$ and $\theta$ satisfy the isentropic relation up to $0\left(\alpha^{1 / 3}\right)$. This implies that the entropy variation, if any, across this region must be of order at least $\alpha^{2 / 3}$.

It should be pointed out here that Eq. (4.17) fails to be a good approximation for $\eta>O\left(\alpha^{2 / 3}\right)$. Consequently the transonic solution, which approaches the asymptote given by Eq. (4.17), cannot be extended beyond the transonic region. It then becomes obvious that the patching of these solutions with the outside solutions must be made at $\eta=O\left(\alpha^{2 / 3}\right)$. This behavior of the present solution is further indicated by the above result that the values of the variables given by the outside expansion at $\eta=O\left(\alpha^{2 / 3}\right)$ fit right into the transonic similarity rule.

5. The solution in the inner supersonic region. The above transonic solution shows that the supersonic branch flow approaches asymptotically to the subsonic branch 


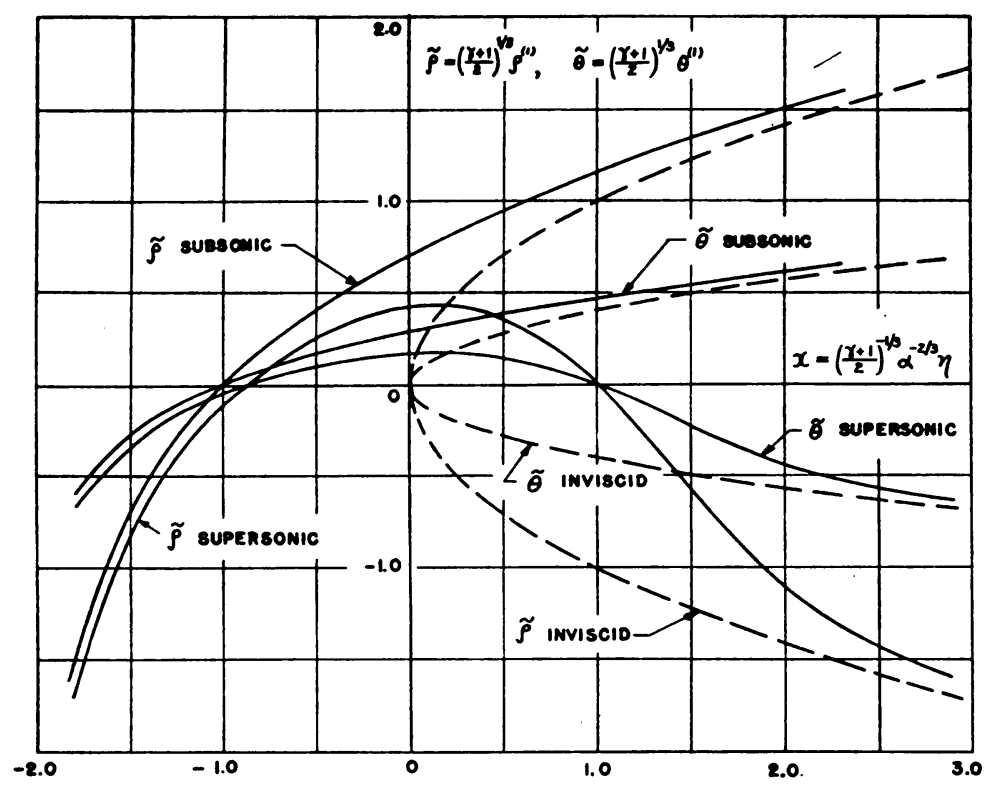

FIG. 7. The thermodynamic variables in the transonic region.

and for $\eta<\eta_{E}$ both branches have supersonic local speed. We shall proceed to find the solution for $\eta<\eta_{E}$. Let us consider first the continuation of the subsonic branch. For the sake of convenience, we shall take the point $E$ in Fig. 6 as the boundary such that at $E, w_{E}=1$ and

$\eta_{E} \doteq-\left(\frac{\gamma+1}{2}\right)^{1 / 3} \alpha^{2 / 3}, \quad V_{E} \doteq\left(\frac{\gamma+1}{2}\right)^{-2 / 3} \alpha^{-1 / 3}, \quad\left(\frac{d V}{d w}\right)_{E} \doteq\left(\frac{\gamma+1}{2}\right)^{-1 / 3} \alpha^{-2 / 3}$.

Now rewrite Eq. (2.6) in the following form

$$
\frac{d V}{d w}=\frac{1}{\alpha}\left(1-\frac{1}{w^{2}}\right)+\frac{1-\beta w^{2}}{\alpha w} \frac{1}{V} .
$$

From the properties of the solution of this equation (see Sec. 2.2) we recall that for $\eta\left\langle\eta_{E}, V\right\rangle V_{E}$, that is, in this region $V$ is at least of $0\left(\alpha^{-1 / 3}\right)$. It then seems convenient to adopt an iteration method to approximate the solution for $\eta<\eta_{E}$. As the first iteration, we neglect the second term on the right hand side of (5.2) and integrate, using (5.1), to obtain

$$
V=\frac{(w-1)^{2}}{\alpha w}+\left(\frac{\gamma+1}{2}\right)^{-2 / 3} \alpha^{-1 / 3}
$$

Substituting this value of $V$ into the right hand side of Eq. (5.2) and integrating twice, using again Eq. (5.1), we obtain, as the second iteration, the following asymptotic result, for $\alpha$ small, valid for $w>1$,

$$
\eta=\eta_{B}-\left(\frac{\gamma+1}{2}\right)^{1 / 3} \alpha^{2 / 3} \arctan \left[\frac{2(w-1)}{w+1}\left(\frac{\gamma+1}{2}\right)^{1 / 3} \alpha^{-1 / 3}\right]+0(\alpha \log \alpha) .
$$


This solution is in good agreement with the value given by Eq. (4.16) for $\eta_{E}<\eta<\eta_{F}$. It can be seen at once that as $w \rightarrow \beta^{-1 / 2}$ (the maximum velocity at which $\theta=0$ ), $\eta$ approaches its smallest value $\eta_{m}$, say, where

$$
\eta_{m}-\eta_{E} \doteq-1.6[(\gamma+1) / 2]^{1 / 3} \alpha^{2 / 3} .
$$

Thus we see that the flow supposedly terminates itself at a distance of $0\left(\alpha^{2 / 3}\right)$ to the inner side of $\eta=0$, beyond which there is no solution to our present system of equations. If one were to investigate further the possibility that one could still obtain a solution of physical reality for $\eta<\eta_{m}$, one would face some rather dubious situations. For instance, near $\eta=\eta_{m}$, the density, temperature and pressure all beeome so low that the validity of the equation of state for a perfect gas (1.4) is questionable. Besides, the fact that the viscous stresses reach the magnitude of the fluid pressure near $\eta=\eta_{m}$ sets a likely limit on the applicability of the Navier-Stokes equation (1.1) and also raises a question as to whether Burnett's higher viscous terms (Ref. [11], p. 271) should be employed to overcome the present difficulty. Of course, it would seem plausible to continue our solution further inward by assigning appropriate values to the arbitrary constant $C$ in (2.16). Nevertheless, it is still impossible to bring the flow to $\eta=-\infty(r=0)$ on account of the singularity that $\rho u \sim r^{-1}$ near $r=0$ (see Eq. 1.3). To clarify these rather vague points is beyond the scope of this paper, although such clarification is certainly desirable.

6. The entropy variation. We define $S$ to be the specific entropy,

$$
T d S=C p d T-\rho^{-1} d p
$$

then the energy equation (1.2) can be written as

$$
\rho u T \frac{d S}{d r}=\operatorname{div}(\lambda \operatorname{grad} T)+\Phi,
$$

where $\Phi$ is the viscous dissipation function, which in this case is,

$$
\Phi=\frac{2}{3}\left(\mu^{\prime}+2 \mu\right)\left[\left(\frac{d u}{d r}\right)^{2}+\left(\frac{u}{r}\right)^{2}\right]+\frac{4}{3}\left(\mu^{\prime}-\mu\right) \frac{u}{r} \frac{d u}{d r} .
$$

The above definition of $S$ of a fluid element is clearly for an open system since the heat exchange by conduction, and hence a net flow of entropy, occurs with the neighboring elements. Thus Eq. (6.2) merely expresses the energy balance, in terms of $S$, of a fluid element-a system not isolated, in the thermodynamic sense, from its surroundings. The analysis of formulating the second law of thermodynamics for the fluid flow case by making the system closed has been investigated in some detail by Tolman and Fine [12] and discussed later by Curtiss and Hirschfelder [13] from the point of view of statistical mechanics. Their idea is, in essence, to state that the change $\Delta S$ in the entropy of a system should consist not only of the net increase in entropy produced by irreversible processes taking place inside the system, but also of the entropy carried into the system, due to conduction of heat energy, equal to div $\left[\lambda T^{-1} \operatorname{grad} T\right]$ per unit volume. Following Tolman's notation, we may thus write

$$
\rho\left(\frac{D S}{D t}\right)=\rho\left(\frac{D S}{D t}\right)_{\mathrm{irr} .}+\operatorname{div}\left(\frac{\lambda}{T} \operatorname{grad} T\right) .
$$


In the present case, Eq. (6.4), after combining with (6.2), becomes

$$
\rho u T\left(\frac{d S}{d r}\right)_{\mathrm{irr} .}=\frac{\lambda}{T}(\operatorname{grad} T)^{2}+\Phi .
$$

As we are only interested in the qualitative features of our later results, we simplify these equations by using the assumptions:

$$
\mu^{*}=\mu / \mu_{1}=1, \quad \mu^{\prime}=0, \quad C_{p}, C .=\text { constant }, \quad \sigma=3 / 4 .
$$

Then the nondimensional form of Eqs. (6.2) and (6.5) are respectively

$$
\begin{gathered}
-\frac{\theta}{\gamma-1} \frac{d s}{d \eta}=\frac{4 \gamma}{3} \alpha^{*}\left\{\frac{1}{\gamma-1} \frac{d^{2} \theta}{d \eta^{2}}+\left(\frac{d w}{d \eta}\right)^{2}-w \frac{d w}{d \eta}+w^{2}\right\} \\
-\frac{\theta}{\gamma-1}\left(\frac{d s}{d \eta}\right)_{\mathrm{irr} .}=\frac{4 \gamma}{3} \alpha^{*}\left\{\frac{\theta^{-1}}{\gamma-1}\left(\frac{d \theta}{d \eta}\right)^{2}+\left(\frac{d w}{d \eta}\right)^{2}-w \frac{d w}{d \eta}+w^{2}\right\}
\end{gathered}
$$

where

$$
s=S / C
$$

Though the sign of the terms on the right hand side of (6.7) is in general indefinite, the value of the right hand side terms of $(6.8)$ is, however, positive definite. Therefore $(s)_{\mathrm{irr}}$ increases monotonically along the fluid flow, as predicted by the second law for a closed system. Subtracting (6.8) from (6.7), we obtain an equation which can be integrated to yield

$$
s_{\mathrm{irr} .}=s+\frac{4 \gamma}{3} \alpha^{*} \frac{d \log \theta}{d \eta},
$$

where the constant of integration is so chosen that both $s$ and $s_{\text {irr }}$ tend to $s_{0}$ as $\eta \rightarrow \infty$, $s_{0}$ being arbitrary.

In order to see that $s$ of the shock type flow reaches a maximum near $w=1$, we substitute Eq. (2.2) with $E=0$ into (6.7) and obtain

$$
\frac{\theta}{\gamma-1} \frac{d s}{d \eta}=\frac{4 \gamma}{3} \alpha^{*} w\left(\frac{d^{2} w}{d \eta^{2}}+\frac{d w}{d \eta}-w\right) \text {. }
$$

This equation shows that for $\eta$ outside the transonic region, the variation in $s$ is at most of $0(\alpha)$. Within the transonic region, $d^{2} w / d \eta^{2}$, being of $0\left(\alpha^{-1}\right)$, overwhelms the rest of the terms in the bracket and hence (6.11) reduces to

$$
\frac{\theta}{\gamma-1} \frac{d s}{d \eta}=\frac{4 \gamma}{3} \alpha^{*} w \frac{d^{2} w}{d \eta^{2}}\left(1+0\left(\alpha^{2 / 3}\right)\right) \text {. }
$$

It then follows that $s$ assumes its maximum value at the point where the curvature of the $w=w(\eta)$ curve vanishes $\left(d^{2} w / d \eta^{2}=0\right.$ at point $G$ in Fig. 6 and at this point $d^{2} s / d \eta^{2}$ is less than zero). However, from (6.10), the quantity $s+\left(4 \gamma \alpha^{*} / 3\right) d \log \theta / d \eta$ does not have an extremum in the entire flow region. The above result is very much the same as that of a plane shock, as it can be shown that the velocity has a point of inflection at sonic speed where the entropy is also a maximum. 
Integrating Eq. (6.11) with the aid of Eqs. (3.1) and (3.2) under condition (6.6), we obtain

where

$$
s-s_{0}=\log \left[\theta\left(w r^{*}\right)^{(\gamma-1)}\right]=\log \theta+(\gamma-1)(\log w+\eta),
$$

$$
s_{0}=\log \left(p_{0} \rho_{0}^{-\gamma}\right),
$$

so that $s \rightarrow s_{0}$ as $r \rightarrow \infty$. This equation is actually the definition of $s$ usually given for a perfect gas. Substitution of the solution (3.12) into (6.13) shows that

$$
\Delta s \sim 0(\alpha) \quad \text { for } \quad \eta>0(1) .
$$

Within and around the transonic region, we substitute the solution (4.1)-(4.3) into (6.13) and simplify the expansion, then we obtain

$$
s-s_{0}=\alpha^{2 / 8}(\gamma-1)[(\gamma+1) / 2]^{1 / 3}\left(x-y^{2}\right)+0(\alpha),
$$

where $x$ and $y$ are defined by Eq. (4.9). The value of $y=y(x)$ is given by Eqs. (4.14), (4.16) and also plotted in Fig. 6. Equation (6.15) is consistent with the fact that $s=s_{0}=$ constant along the inviscid solution $y^{2}=x$. The variation in $s$ along supersonic and subsonic branches of our solution follows directly from the data shown in Fig. 6 . The result is plotted in Fig. 8. As $\eta$ decreases along the supersonic branch, the entropy $s$

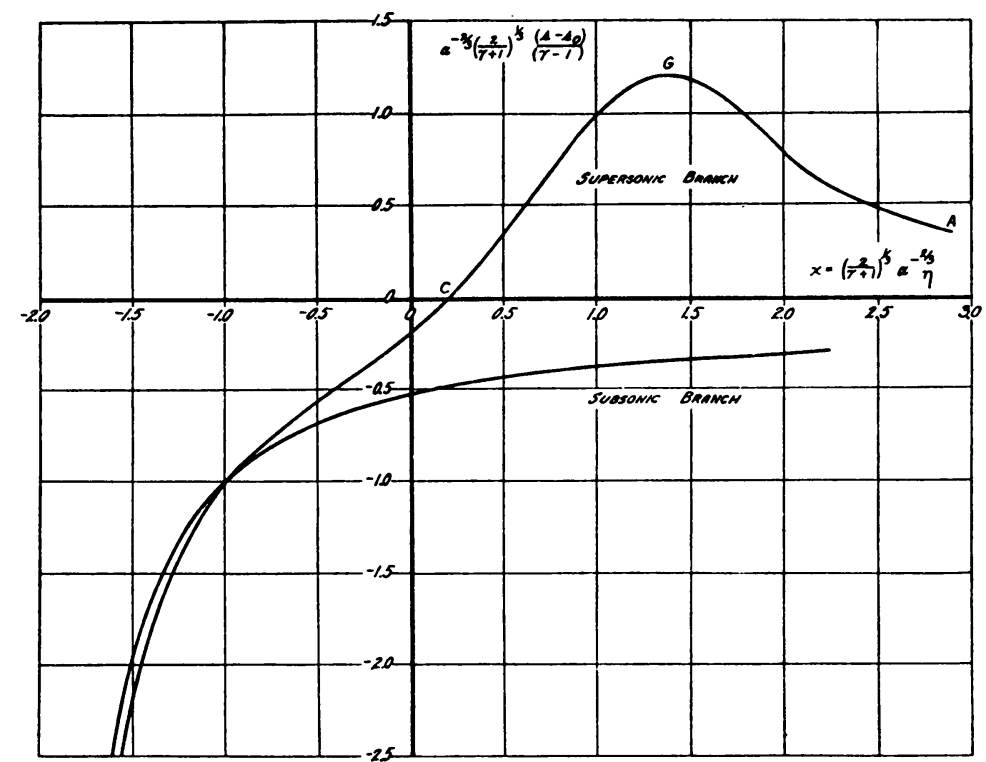

FIG. 8. The entropy variation across the transonic region.

first increases until it reaches the maximum $s_{0}+1.2 \alpha^{2 / 3}(\gamma-1)[(\gamma+1) / 2]^{1 / 3}$ at point $G$, then decreases and later assumes once again the value $s_{0}$ (the value of $s$ at $\eta=\infty)$ at point $C$ where $w^{(1)}$ is minimum. After that $s$ decreases rapidly with further decrease in $\eta$ and eventually tend to $-\infty$ as the flow solution terminates. On the subsonic branch, $s$ decreases monotonically with decreasing $\eta$. However, by substituting Eqs. (6.15) and (4.3) into (6.10), it can easily be shown that $\left(s_{1 \mathrm{rr}}\right.$ ) increases monotonically 
with decreasing $\eta$ and the variation in $\left(s_{\mathrm{irr} .}\right)$ is of order $0(\alpha)$. Consequently, the result that $s \rightarrow-\infty$ as $\eta \rightarrow \eta_{\min }$ can be explained by visualizing from Eq. (6.10) that $d(\log \theta) / d \eta$ decreases beyond all bounds as $\eta \rightarrow \eta_{\min }$. Physically, this probably implies that the flow is rather far from its equilibrium condition due to the large velocity gradient, inducing a rapid decrease in temperature, which even the important heat conduction in this region cannot compensate.

\section{REFERENCES}

1. Y. T. Wu, Two dimensional sink flow of a viscous, heat-conducting compressible fluid; cylindrical shock waves, Hydrodỵnamics Laboratory Report No. 21-16, California Institute of Technology, California (1954)

2. F. Ringleb, Exact solutions of the differential equations of an adiabatic gas flow, Ministry of Aircraft Production, Great Britain, R. T. P. Translation 1609 (1942)

3. R. V. Hess, $A$ solution of the Navier-Stokes equation for source and sink flows of a viscous, heat-conducting compressible fluid, NACA TN 2630 (1952)

4. A. Sakurai, On the theory of cylindrical shock wave, J. Phys. Soc., Japan (4-6) 4, 199-202 (1949)

5. H. C. Levey, Two dimensional source flow of a viscous fluid, Quart. Appl. Math. 7, No. 1, 25-48 (1954)

6. J. G. Kirkwood, F. P. Buff, and M. S. Green, The statistical mechanical theory of transport processes. III. The coefficients of shear and bulk viscosity of liquids, J. Chem. Phys. 17, 988 (1949)

7. E. Kemke, Differentialgleichungen reeller Funktionen, Chelsea Publishing Co., New York, 1947

8. J. J. Stoker, Nonlinear vibrations, Interscience Publishers, New York, 1950

9. Tables of Bessel functions of fractional order, Vol. I, II, National Bureau of Standards, Columbia University Press, 1949

10. R. von Mises, On the thickness of a steady shock wave, J. Aeronaut. Sci. 17, No. 9, 551-554 (1950)

11. S. Chapman and T. G. Cowling, The mathematical theory of non-uniform gases, Cambridge University Press, 1939

12. R. C. Tolman and P. C. Fine, On the irreversible production of entropy, Rev. Modern Phys. 20, No. 1, 51-77 (1948)

13. C. F. Curtiss and J. O. Hirschfelder, The thermodynamics of flow systems, J. Chem. Phys. 18, No. 2, 171-173 (1950) 